\title{
Nonlinear Vibration Behavior of Sandwich Beams with Entangled Fiber Core Material
}

\author{
Elsa Piollet ${ }^{1}$, Guilhem Michon ${ }^{1}$, and Dominique Poquillon ${ }^{2}$ \\ ${ }^{1}$ Université de Toulouse, ICA (Institut Clément Ader), ISAE, F-31055, Toulouse, FRANCE \\ ${ }^{2}$ Université de Toulouse, CIRIMAT, INP-ENSIACET, F-31030, Toulouse, FRANCE
}

\begin{abstract}
In this paper, the use of entangled cross-linked fibers as core material in vibrating sandwich beams is investigated. The aim is to analyze the effect of this specific core material in terms of damping. The dynamic shear properties of the material are first studied experimentally. The shear modulus is shown to decrease with increasing shear strain amplitude at low shear strains. To include an amplitude dependency of the core material properties in the sandwich beam behavior, an analytical model is proposed. The equations of motion are derived using Lagrange's equations. The shearing of the core is introduced in the equations through the use of virtual work to allow any relationship between shear stress and shear strain, including damping and nonlinearities. Experimental tests are carried out on sandwich beams with entangled fiber core material. The Frequency Response Function obtained exhibits decreasing resonant frequency and peak amplitude with increasing load amplitude. This softening behavior is consistent with the decreasing shear modulus. The proposed model is used take into account the softening nonlinearity. The FRF is reproduced with a linearly decreasing shear modulus and linearly increasing loss factor.
\end{abstract}

\section{Introduction}

Sandwich panels are widely used in aerospace and automotive structures. They are made of a core bonded to two stiff facesheets. By separating the two facesheets with the core, the bending stiffness is increased as compared to a single-material structure of the same weight. While this high stiffness-to-weight ratio is a strong advantage from a static point of view, it also makes the structures prone to transmit vibrations. In aerospace and automotive fields, unwanted vibrations can lead to user discomfort or damage to the structures, and vibration damping is thus a strong concern. The theory of vibration of damped three-layer beams and panels was considered by a number of authors in the past 50 years. Kerwin [1] analysed the use of constrained layer to damp flexural waves. DiTaranto [2] and Mead and Markus [3] proposed a model for sandwich beams with arbitrary conditions and a viscoelastic core, and set the hypotheses that are still in use in the literature. The use of the energy principle (Hamilton's principle or Lagrange's equations) to derive the equations of motion was introduced by Rao [4].

The most commonly used core material is honeycomb, which does not provide much damping. Control must then be added through either active or passive damping. Active damping consists in the use of sensors and actuators, which need energy input. Passive damping consists in using adapted materials to increase the overall damping of the structure. A layer of viscoelastic material can be added to the structure, either by covering the structure with a constrained or unconstrained viscoelastic layer, or by including the viscoelatic layer between the facesheet and the core [5]. Another possibility lies in considering new core materials to replace totally or partially the classical honeycomb material, as by combining honeycomb and viscoelastic material for example [6]. This paper studies the flexural vibration of sandwich beams with a recently introduced core material. Mezeix et al. [7] developed a new core material made of short fibers of glass, carbon or aramid, entangled and cross-linked with epoxy resin. Some 




Figure 1: Entangled cross-linked fiber material: shear sample.

vibrations experiments have been carried out by Mezeix [8] and Shahdin [9], but this is the first study focused on the vibration properties and modeling of sandwich beams with this core material.

Previous models of sandwich beams considered frequency dependent materials, or geometrical nonlinearities, but no inclusion of amplitude dependent material behavior could be found in the literature.

In this paper, the amplitude-dependency of the dynamic shear properties of the entangled cross-linked fiber material is observed experimentally. An analytical model of the flexural vibration of sandwich beams is derived using Lagrange's equations to include core material nonlinearity. A sandwich beam with entangled cross-linked fibers as core material is studied experimentally. The proposed model is used to reproduce the observed frequency response, and analytical and experimental results are compared.

\section{Entangled cross-linked fiber material description}

After studies of Poquillon et al. [10] on entangled fibers, Mezeix et al. [7] introduced a material in which short entangled fibers are cross-linked with epoxy resin. Contrarily to most architectured materials, entangled crosslinked fibers have no periodicity: the fiber orientations are randomly distributed.

Different types of fibers can be used and previous studies considered carbon, aramid and glass fibers $[9,7]$. In this paper, the material is made with carbon fibers which are commonly used in aeronautical applications due to their high performances in terms of Young's modulus and tensile strength. The fibers consist in a yarn of stranded carbon filaments. The diameter of the filaments is $7 \mu \mathrm{m}$ with a Young's modulus of $240 \mathrm{GPa}$.

The fibers are first cut to a length of $40 \mathrm{~mm}$. To achieve a low density material, the filaments of the yarn must be separated. A blower room is used to obtain the separation and to entangle the fibers, with an air flux of 4 bar pressure.

Then, the entangled fibers are cross-linked with epoxy resin. Epoxy resin is chosen because of the common use of carbon/epoxy prepreg as facesheets in aeronautical sandwich structures. A paint spray gun is used to spray the resin.

Finally, the fiber network is compressed to fit in the mold and polymerized. Figure 1 shows a sample of the material after polymerization.

The volume fraction of the material is defined as the ratio of the entanglement density (before cross-linking) to the density of the bulk material:

$$
f=\frac{\rho_{\text {entanglement }}}{\rho_{\text {bulk }}}
$$

In the present paper, the density of the entanglement is $150 \mathrm{~kg} / \mathrm{m}^{3}$. The density of the carbon is $1756 \mathrm{~kg} / \mathrm{m}^{3}$, 




Figure 2: Experimental set-up for dynamic shear test.

so the volume fraction is $8.5 \%$. The epoxy cross-links of a sample represent a density of $30 \mathrm{~kg} / \mathrm{m}^{3}$. Thus, the final material has a total density of $180 \mathrm{~kg} / \mathrm{m}^{3}$.

\section{Material identification}

\subsection{Set-up and samples}

This paper focuses on the use of entangled cross-linked fibers as sandwich core material in flexural vibration. As in a large part of the literature $[2,3,11]$, the core material is supposed to be deformed only in shear. The material is thus characterized in shear only.

The samples have a thickness of $20 \mathrm{~mm}$, a length of $60 \mathrm{~mm}$ and a width of $40 \mathrm{~mm}$. One sample is shown on Fig. 1. The samples are bonded to $3 \mathrm{~mm}$ thick aluminum plates on both sides. To ensure that the applied deformation is in shear only, two samples are tested together. A vertical excitation is applied between the two samples by a $100 \mathrm{~N}$ shaker. The vertical displacement of the central aluminum plates is measured with an inductive displacement sensor and the force is measured with a piezoelectric load cell located at the interface between the shaker and the aluminum plates. The set-up for dynamic shear testing is presented on Fig. 2.

The shear strain $\gamma$ and shear stress $\tau$ are related to the measured displacement $u$ and load $F$ as follows:

$$
\begin{gathered}
\gamma=\frac{u}{h} \\
\tau=\frac{F}{S_{2}}
\end{gathered}
$$

where $h=20 \mathrm{~mm}$ is the thickness of the sample and $S_{2}=2 \times 40 \mathrm{~mm} \times 60 \mathrm{~mm}$ the area through which the load is applied, i.e. the bonded area between the two samples and the central aluminum plates.

\subsection{Viscoelastic model}

The most classical way to model material including stiffness and damping is to use a viscoelastic modeling, as described by Nashif et al. [12] and Lakes [13] among others.

In case of harmonic loading, the expression of the oscillatory shear strain is:

$$
\gamma=\gamma_{0} \sin (\omega t)
$$

Then in the case of linear viscoelastic behavior, the resulting shear stress is also sinusoidal with a phase lag $\phi$ :

$$
\tau=\tau_{0} \sin (\omega t+\phi)
$$




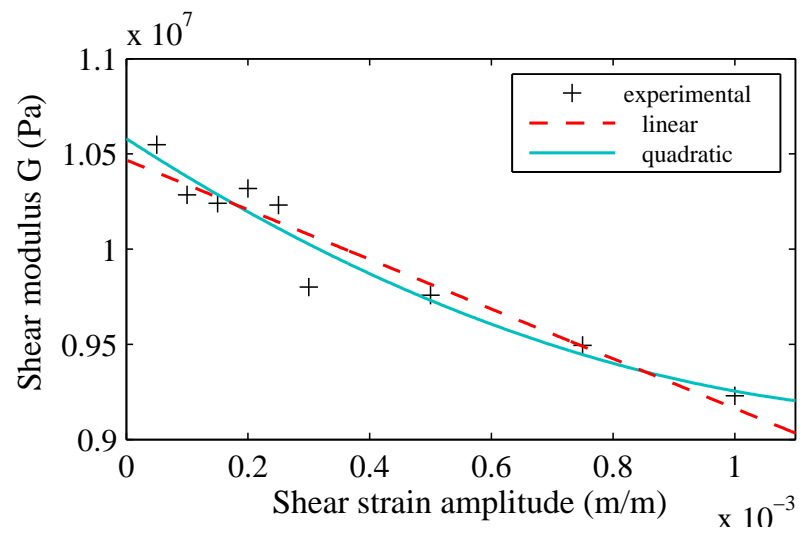

Figure 3: Evolution of shear modulus with the shear strain amplitude.

which is related to the shear strain amplitude by the expression:

$$
\tau=G^{\prime} \gamma_{0} \sin (\omega t)+G^{\prime \prime} \gamma_{0} \cos (\omega t)
$$

or

$$
\tau=G^{\prime} \gamma+\frac{G^{\prime \prime}}{\omega} \dot{\gamma}
$$

where $G^{\prime \prime}=G^{\prime} \tan (\phi)$ and ${ }^{*}=\frac{d}{d t}$. $G^{\prime}$ is the storage modulus, $G^{\prime \prime}$ is the loss modulus, and $\eta=\tan (\phi)$ is the loss factor.

These expressions are often used in a complex form with $\gamma=\gamma_{0} \exp (i \omega t)$. Then $\tau=G^{*} \gamma$ with $G^{*}=G^{\prime}+i G^{\prime \prime}=G^{\prime}(1+i \eta)$, $i=\sqrt{-1}$.

Experimentally, $G^{\prime}$ and $\eta$ are measured from stress-strain loops by using the following relationships:

$$
\begin{gathered}
G^{\prime}=\frac{\tau_{\max }}{\gamma_{\max }} \\
\eta=\frac{\tau(\gamma=0)}{\tau_{\max }}
\end{gathered}
$$

In small deformations, viscoelastic models are generally used with $\mathrm{G}$ and $\eta$ being frequency-dependent but amplitude-independent. Stress-strain loops are then elliptic. However, these models are also used as approximations to describe an amplitude-dependent behavior by defining the storage modulus and loss factor as functions of the amplitude, see [14] for example. When the nonlinearity increases, the stress-strain loop is no longer elliptic and other models must be used.

\subsection{Parameter identification}

Measurements are made at $20 \mathrm{~Hz}$ to stay in a quasi-static configuration. In this paper, the focus is on the amplitude dependency. The dissipation is expected to come mainly from friction between fibers, which is a frequencyindependent phenomenon. The hypothesis of amplitude-dependent and frequency-independent material is consistent with the work of Pritz on other fibrous materials such as mineral wool $[15,14]$.

The range of study is from $5 \cdot 10^{-5}$ to $1.10^{-3}$ for $\gamma$, corresponding to $1.10^{-3} \mathrm{~mm}$ to $2.10^{-2} \mathrm{~mm}$ for the vertical displacement $u$.

Figure 3 shows the evolution of the shear modulus with the shear strain amplitude. The modulus descreases with the amplitude. To describe this evolution, a linear relationship is used:

$$
G=1.0 \cdot 10^{7}-1.3 \cdot 10^{9}|\gamma|
$$






Figure 4: Clamped-free sandwich beam and lumped parameter electrodynamic shaker.



Figure 5: A section of a sandwich beam in deformed and undeformed configurations.

For $\gamma=5.10^{-4}$ and $\gamma=1.10^{-3}$, a loss factor of $\eta=0.06$ is measured. At lower amplitudes, the loss factor is too small to be measured.

\section{Sandwich beam model}

\subsection{Geometry and hypotheses}

The system studied is a clamped-free sandwich beam with an electrodynamic shaker modeled by its moving mass and stiffness as shown on Fig. 4.

The sandwich beam is of length L and width B. It is composed of three layers of thickness $h_{\beta}$ and density $\rho_{\beta}$ with $\beta=\mathrm{t}$, $\mathrm{b}$ or $\mathrm{c}$, respectively, for the top layer, bottom layer and core layer. The beam is clamped at $x=0$, and free at $x=L$.

The theoretical developments presented here are based on assumptions made by Mead and Markus [3] and commonly accepted in the literature:

- Displacements are small and occur in the $x z$ plane only.

- Laminates are considered purely elastic, with Young moduli $E_{\mathrm{t}}$ and $E_{\mathrm{b}}$. Laminates are treated as EulerBernoulli beams: shear strains and rotary inertia are neglected. 
- Transverse direct strains in both core and face-plates are also neglected: for a given position $x$, transverse displacement $w$ in the $z$ direction is identical in the three layers, which are assumed to be perfectly bonded. Bonding layer thickness is considered to be null.

- Shear stress and shear strain are constant across the depth of the core.

There is no assumption made on the core material behavior at this point.

The geometry of the beam and notations taken are illustrated on Fig. 5. The longitudinal $\mathrm{x}$-wise displacements of the mid-planes of the face-plates are $u_{\mathrm{t}}(x, t)$ and $u_{\mathrm{b}}(x, t)$. The transverse $\mathrm{z}$-wise displacement of the system is $w(x, t)$.

The shear strain $\gamma$ in the core is related to the displacements as follows:

$$
\gamma=\frac{u_{\mathrm{t}}-u_{\mathrm{b}}}{h_{\mathrm{c}}}+\frac{d}{h_{\mathrm{c}}} \frac{\partial w}{\partial x}
$$

where

$$
d=h_{\mathrm{c}}+\frac{1}{2}\left(h_{\mathrm{t}}+h_{\mathrm{b}}\right)
$$

and the longitudinal displacement at the middle of the core is given by:

$$
u_{\mathrm{c}}=\frac{1}{2}\left(u_{\mathrm{t}}+u_{\mathrm{b}}+\frac{h_{\mathrm{t}}-h_{\mathrm{b}}}{2} \frac{\partial w}{\partial x}\right)
$$

\subsection{Energies and work}

\subsubsection{Kinetic energy of the system.}

The kinetic energy of the system results from the transverse and longitudinal motion of the three layers, neglecting rotational kinetic energy, and the moving mass of the shaker (subscript s):

$$
T=T_{\mathrm{t}}+T_{\mathrm{b}}+T_{\mathrm{c}}+T_{\mathrm{s}}
$$

where

$$
T_{\beta}=\frac{1}{2} \rho_{\beta} h_{\beta} B \int_{0}^{L}\left[\left(\frac{\partial u_{\beta}}{\partial t}\right)^{2}+\left(\frac{\partial w}{\partial t}\right)^{2}\right] d x
$$

for $\beta=t, b$ and $c$, and

$$
T_{\mathrm{s}}=\frac{1}{2} m_{\mathrm{s}}\left(\frac{\partial w}{\partial t}\left(x_{f}\right)\right)^{2}
$$

\subsubsection{Potential energy of the system.}

The potential energy of the system is due to the deformation of the two elastic laminates and the deformation of the shaker's spring:

$$
V=V_{\mathrm{t}}+V_{\mathrm{b}}+V_{\mathrm{s}}
$$

where

$$
V_{\alpha}=\frac{1}{2} \int_{L_{\alpha}}\left[E_{\alpha} A_{\alpha}\left(\frac{\partial u_{\alpha}}{\partial x}\right)^{2}+E_{\alpha} I_{\alpha}\left(\frac{\partial^{2} w}{\partial x^{2}}\right)^{2}\right] d x
$$

for $\alpha=t$ and $b$, where $E_{\alpha}$ is the Young's modulus of the laminate, $A_{\alpha}=h_{\alpha} B$ the cross-sectional area and $I_{\alpha}$ the second moment of area, and

$$
V_{\mathrm{s}}=\frac{1}{2} k_{\mathrm{s}} w^{2}
$$




\subsubsection{Virtual work in the core.}

To include any shear behavior of the core, and in particular nonconservative and nonlinear behavior, the contribution of the core to the energy of the system is written as the virtual work of the shear stress:

$$
\delta W_{\mathrm{c}}=\frac{1}{2} \int_{V} \tau \delta \gamma d V
$$

By using the hypothesis of a constant shear stress through the thickness and width of the beam, the expression is simplified to:

$$
\delta W_{\mathrm{c}}=\frac{1}{2} B h_{\mathrm{c}} \int_{0}^{L} \tau \delta \gamma d x
$$

\subsubsection{Virtual work due to the external load.}

The virtual work of an external transverse load $f(x, t)$ is:

$$
\delta W_{f}=\int_{0}^{L} f(x, t) \delta w(x, t) d x
$$

\subsubsection{Assumed modes.}

In order to write Lagrange's equations of motion, generalized coordinates need to be defined. The assumed mode method is used to discretize the continuous system. Three sets of admissible functions are used to discretize the transverse displacement of the beam and the two longitudinal displacements of the laminates. The use of a third set of admissible functions for $u_{\mathrm{t}}$ was introduced by Cai et al. [11]. The time and space dependency are separated and a vector notation is used to obtain the following relationships:

$$
\begin{gathered}
w(x, t)=\sum_{i}^{n_{w}} W_{i}(x) \psi_{i}(t)=\mathbf{W}^{\mathrm{T}} \psi \\
u_{\mathrm{b}}(x, t)=\sum_{j}^{n_{\mathrm{b}}} U_{b, j}(x) \xi_{b, j}(t)=\mathbf{U}_{\mathrm{b}}^{\mathrm{T}} \xi_{\mathrm{b}} \\
u_{\mathrm{t}}(x, t)=\sum_{k}^{n_{\mathrm{t}}} U_{t, k}(x) \xi_{t, k}(t)=\mathbf{U}_{\mathrm{t}}^{\mathrm{T}} \xi_{\mathrm{t}}
\end{gathered}
$$

The displacement functions $W_{i}, U_{b, j}$ and $U_{t, k}$ are sets of admissible functions, $\psi_{i}, \xi_{b, j}$ and $\xi_{t, k}$ are the new generalized coordinates, and $n_{w}, n_{\mathrm{b}}$ and $n_{\mathrm{t}}$ are the number of modes selected respectively for the transverse motion of the system, the longitudinal motion of the bottom layer and the longitudinal motion of the top layer. If $n \rightarrow \infty$ for each of the different displacements, the response is considered to be exact. Superscript T denotes the transpose of a vector.

Classical mode shape functions [16] are chosen as admissible functions, as they satisfy all geometric boundary conditions. In this study, the three layers are clamped-free beams. For transverse displacement of a clamped-free beam the corresponding mode shape function is:

$$
W_{i}(x)=\cosh \left(\lambda_{i} x\right)-\cos \left(\lambda_{i} x\right)-\sigma_{i}\left[\sinh \left(\lambda_{i} x\right)-\sin \left(\lambda_{i} x\right)\right]
$$

where

$$
\sigma_{i}=\frac{\sinh \left(\lambda_{i} L\right)-\sin \left(\lambda_{i} L\right)}{\cosh \left(\lambda_{i} L\right)+\cos \left(\lambda_{i} L\right)}
$$

and $\lambda_{1} L=1.875, \lambda_{2} L=4.694, \lambda_{i} L=(i-0.5) \pi$ for $i=3, \ldots, n_{w}$ where $L$ is the length of the beam. 
For longitudinal displacement in a clamped-free configuration:

$$
U_{t, i}(x)=U_{b, i}(x)=\sin \frac{\pi(2 i-1) x}{2 L}
$$

for $i=1,2,3 \ldots n_{u_{\mathrm{t}}}$ or $n_{u_{\mathrm{b}}}$.

Substituting Eqn. (23)-(25) in the kinetic and potential energy expressions and using Eqn. (13) for the expression of core longitudinal displacement lead to:

$$
T=\frac{1}{2}\left\{\begin{array}{c}
\dot{\psi} \\
\dot{\xi_{\mathrm{b}}} \\
\dot{\dot{\xi}_{\mathrm{t}}}
\end{array}\right\}^{\mathrm{T}}\left[\begin{array}{lll}
\mathbf{M}_{w w} & \mathbf{M}_{u_{\mathrm{b}} w}^{\mathrm{T}} & \mathbf{M}_{u_{\mathrm{t}} w}^{\mathrm{T}} \\
\mathbf{M}_{u_{\mathrm{b}} w} & \mathbf{M}_{u_{\mathrm{b}} u_{\mathrm{b}}} & \mathbf{M}_{u_{\mathrm{t}} u_{\mathrm{b}}}^{\mathrm{t}} \\
\mathbf{M}_{u_{\mathrm{t}} w} & \mathbf{M}_{u_{\mathrm{t}} u_{\mathrm{b}}} & \mathbf{M}_{u_{\mathrm{t}} u_{\mathrm{t}}}
\end{array}\right]\left\{\begin{array}{c}
\dot{\psi} \\
\dot{\xi_{\mathrm{b}}} \\
\dot{\dot{\xi}_{\mathrm{t}}}
\end{array}\right\}
$$

and

$$
V=\frac{1}{2}\left\{\begin{array}{c}
\psi \\
\xi_{\mathrm{b}} \\
\xi_{\mathrm{t}}
\end{array}\right\}^{\mathrm{T}}\left[\begin{array}{ccc}
\mathbf{K}_{w w} & 0 & 0 \\
0 & \mathbf{K}_{u_{\mathrm{b}} u_{\mathrm{b}}} & 0 \\
0 & 0 & \mathbf{K}_{u_{\mathrm{t}} u_{\mathrm{t}}}
\end{array}\right]\left\{\begin{array}{c}
\psi \\
\xi_{\mathrm{b}} \\
\xi_{\mathrm{t}}
\end{array}\right\}
$$

The submatrices are presented in Annexe A.

\subsection{Equation of motion}

\subsubsection{Lagrange's equations.}

Lagrange's equations can be written as follows:

$$
\frac{d}{d t}\left(\frac{\partial L}{\partial \dot{q}_{k}}\right)-\frac{\partial L}{\partial q_{k}}=Q_{k}
$$

where $k=1,2, \ldots, N_{\text {dof }}, L=T-V$ is the Lagrangian and $Q_{k}$ represents the generalized forces for the $k^{\text {th }}$ generalized coordinate, defined as:

$$
Q_{k}=\frac{\delta W}{\delta q_{k}}
$$

For an external force applied locally at $x=x_{f}, f(x, t)=f(t) \delta\left(x-x_{f}\right)$, the expression of the virtual work after discretization is:

$$
\delta W_{f}=\sum_{i}^{n_{w}} \delta \psi_{i}(t) \int_{0}^{L} f(t) \delta\left(x-x_{f}\right) W_{i}(x) d x
$$

Hence, from Eqn. (32), the generalized forces due to the transverse force are:

$$
\mathbf{Q}_{f}=\left\{\begin{array}{c}
f(t) \mathbf{W}_{x=x_{f}} \\
0 \\
0
\end{array}\right\}
$$

For the core, the variation and discretization of Eqn. (11) gives:

$$
\delta \gamma=\frac{1}{h_{\mathrm{c}}}\left(\mathbf{U}_{\mathrm{t}}^{\mathrm{T}} \delta \xi_{\mathrm{t}}-\mathbf{U}_{\mathrm{b}}^{\mathrm{T}} \delta \xi_{\mathrm{b}}\right)+\frac{d}{h_{\mathrm{c}}} \delta \psi\left(\frac{\partial \mathbf{W}}{\partial x}\right)
$$

which is introduced in Eqn. (21) and Eqn. (32) to obtain the generalized force:

$$
\mathbf{Q}_{\mathrm{c}}=\left\{\begin{array}{c}
-B d \int_{0}^{L} \tau \mathbf{W}_{, x} d x \\
B \int_{0}^{L} \tau \mathbf{U}_{\mathrm{b}} d x \\
-B \int_{0}^{L} \tau \mathbf{U}_{\mathrm{t}} d x
\end{array}\right\}
$$




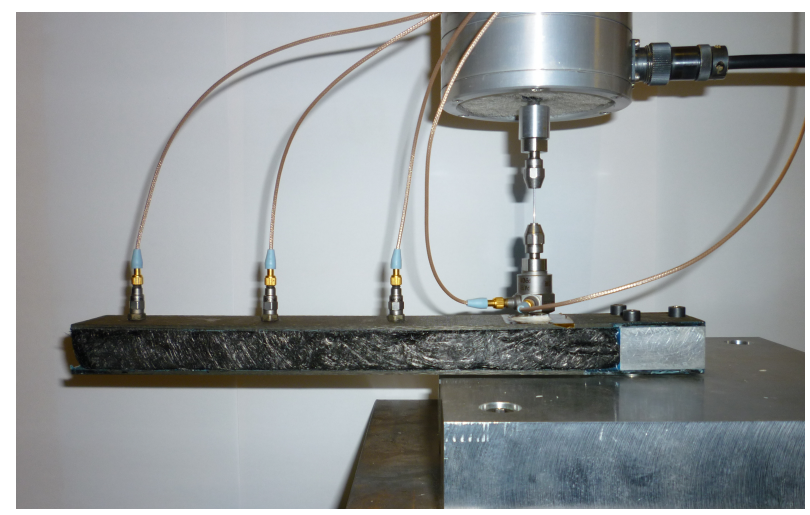

Figure 6: Experimental set-up for flexural vibration.

In the case of viscoelasticity, the expression of $\tau$ given by Eqn. (7) yields:

$$
\mathbf{Q}_{\mathrm{c}}=\left\{\begin{array}{c}
-B d \int_{Q}^{L}\left(G \gamma_{\mathrm{c}}+\frac{G}{\omega} \dot{\gamma}_{\mathrm{c}}\right) \mathbf{W}_{, x} d x \\
B \int_{0}^{L}\left(G \gamma_{\mathrm{c}}+\frac{G}{\omega} \dot{\gamma}_{\mathrm{c}}\right) \mathbf{U}_{\mathrm{b}} d x \\
-B \int_{0}^{L}\left(G \gamma_{\mathrm{c}}+\frac{G}{\omega} \dot{\gamma}_{\mathrm{c}}\right) \mathbf{U}_{\mathrm{t}} d x
\end{array}\right\}
$$

While this expression simplifies when the core is linearly viscoelastic, i.e. G does not depend on $\gamma$, in the case of nonlinear viscoelasticity the expression has to be integrated at each step.

Finally, the equation of motion for the generalized core behavior is:

$$
\begin{array}{r}
{\left[\begin{array}{lll}
\mathbf{M}_{w w} & \mathbf{M}_{u_{\mathrm{b}} w}^{\mathrm{T}} & \mathbf{M}_{u_{\mathrm{t}} w}^{\mathrm{T}} \\
\mathbf{M}_{u_{\mathrm{b}} w} & \mathbf{M}_{u_{\mathrm{b}} u_{\mathrm{b}}} & \mathbf{M}_{u_{\mathrm{t}} u_{\mathrm{b}}}^{\mathrm{T}} \\
\mathbf{M}_{u_{\mathrm{t}} w} & \mathbf{M}_{u_{\mathrm{t}} u_{\mathrm{b}}} & \mathbf{M}_{u_{\mathrm{t}} u_{\mathrm{t}}}
\end{array}\right]\left\{\begin{array}{c}
\ddot{\psi} \\
\ddot{\xi_{\mathrm{b}}} \\
\ddot{\xi_{\mathrm{t}}}
\end{array}\right\}+\left[\begin{array}{ccc}
\mathbf{K}_{w w} & 0 & 0 \\
0 & \mathbf{K}_{u_{\mathrm{b}} u_{\mathrm{b}}} & 0 \\
0 & 0 & \mathbf{K}_{u_{\mathrm{t}} u_{\mathrm{t}}}
\end{array}\right]\left\{\begin{array}{c}
\psi \\
\xi_{\mathrm{b}} \\
\xi_{\mathrm{t}}
\end{array}\right\}} \\
=\left\{\begin{array}{c}
\mathbf{Q}_{c, w}+\mathbf{Q}_{f} \\
\mathbf{Q}_{c, u_{\mathrm{b}}} \\
\mathbf{Q}_{c, u_{\mathrm{t}}}
\end{array}\right\}
\end{array}
$$

\section{$5 \quad$ Experimental study and discussion}

\subsection{Experimental set-up and specimen}

The beam studied has a length $L=264 \mathrm{~mm}$ and width $B=40 \mathrm{~mm}$. The sandwich is symmetrical with core thickness $h_{\mathrm{c}}=20 \mathrm{~mm}$ and facesheet thickness $h_{\mathrm{t}}=h_{\mathrm{b}}=2 \mathrm{~mm}$.

The core is made of entangled carbon fibers with a volume fraction $f=8.5 \%$, cross-linked with epoxy resin for a total density $\rho_{\mathrm{c}}=180 \mathrm{~kg} / \mathrm{m}^{3}$. The facesheets are made of seven plies of prepreg carbon fabric used in the aeronautical industry. The density and Young's modulus are measured experimentally: $\rho_{\mathrm{b}}=\rho_{\mathrm{t}}=1.5 \cdot 10^{3} \mathrm{~kg} / \mathrm{m}^{3}$ and $E_{\mathrm{b}}=E_{\mathrm{t}}=3.26 \cdot 10^{10} \mathrm{~Pa}$.

The beam is clamped at $x=0$ and free at $x=L$. Excitation is applied with an electrodynamic shaker at $x_{f}=37 \mathrm{~mm}$. The excitation is close to the clamped end to minimize coupling between the shaker and the beam. Acceleration is measured at four points on the top of the beam which positions are $x=[37 \mathrm{~mm}, 104 \mathrm{~mm}, 171 \mathrm{~mm}, 238 \mathrm{~mm}]$.

The electrodynamic shaker's moving mass is $m_{\mathrm{s}}=2 \cdot 10^{-2} \mathrm{~kg}$. The stiffness is $k_{\mathrm{s}}=576 \mathrm{~N}$. 
(a) Frequency response function for loads from $0.04 \mathrm{~N}$ to $2 \mathrm{~N}$
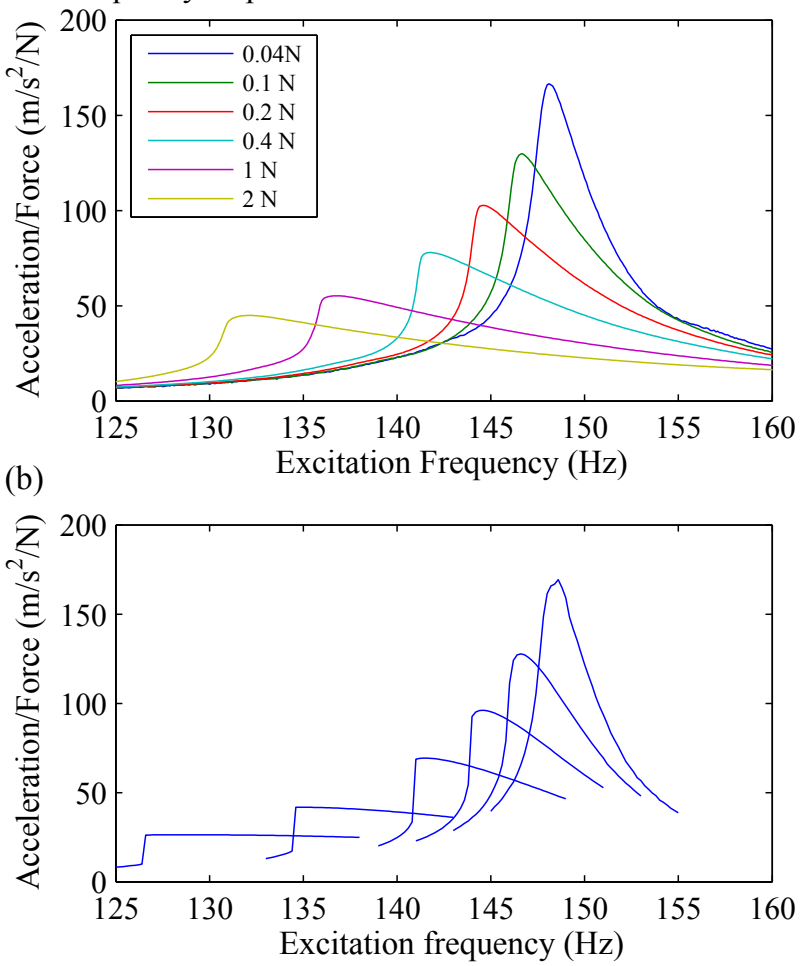

Figure 7: Frequency response function of the sandwich beam with entangled carbon fiber core for different load amplitudes (a) experimental results (b) model for $G=1.05 \cdot 10^{7}-1.5 \cdot 10^{10}|\gamma|$ and $\eta=1.2 \cdot 10^{-2}+6.5 \cdot 10^{2}|\gamma|$.

\subsection{Experimental results}

A sinusoidal load of constant amplitude is applied to the moving mass of the shaker with stepped frequency. Different amplitudes are applied to observe the effect of the amplitude-dependency of the core properties on the overall beam behavior: $F_{0}=[0.04 \mathrm{~N}, 0.1 \mathrm{~N}, 0.2 \mathrm{~N}, 0.4 \mathrm{~N}, 1 \mathrm{~N}, 2 \mathrm{~N}]$.

The resulting Frequency Response Function (FRF) is shown on Fig. 7(a) for the fourth accelerometer, at $x=238 \mathrm{~mm}$. The same behavior is observed for the four accelerometers.

The FRF shows a strong dependency of the resonance peak on the load amplitude. Both the resonance frequency and peak amplitude decrease when the load amplitude increases. Moreover, the peak is asymmetrical.

This softening nonlinear behavior is consistent with the observation made in the first section that the shear modulus decreases with increasing shear strain amplitude.

The asymmetry of the peak prevents the use of a classical loss factor definition. However, the observed decreasing amplitude of the acceleration over load ratio indicates that the loss factor increases with increasing shear strain amplitude. This kind of softening core material can be interesting at higher load amplitudes as compared to classical core materials.

\subsection{Comparison with the model}

To compare the analytical model with the experimental data, Eqn. (38) is integrated in the time domain for different load amplitudes. The focus is made on the first bending mode. The number of modes used is $n_{w}=n_{\mathrm{b}}=n_{\mathrm{t}}=2$. 
For fast computation, the spatial integration for the generalized force $Q$ is made using the midpoint rule:

$$
\int_{0}^{L} f(x) d x \approx \frac{L}{n} \sum_{k=1}^{n} f\left(\frac{k+\frac{1}{2}}{n} L\right)
$$

The present computation is made with $n=4$.

Figure 7(b) shows the obtained FRF for the following storage modulus and loss factor functions:

$$
\begin{gathered}
G=1.05 \cdot 10^{7}-1.5 \cdot 10^{10}|\gamma| \\
\eta=1.2 \cdot 10^{-2}+6.5 \cdot 10^{2}|\gamma|
\end{gathered}
$$

These results show that the proposed model allows to capture the behavior of a sandwich beam with core material nonlinearities. The modeling of $\mathrm{G}$ and $\eta$ by a linear dependency with shear strain amplitude is sufficient to yield the observed behavior.

The parameters can be optimized to fit exactly the measures, but it was not the purpose of this work. The difference in the values between the shear test and the sandwich beam test can be explained by the heterogeneity of the material, that leads to a strong sensitivity to the sample size. The fact that very small strains are experienced inside the sandwich beam (from $10^{-6}$ to $10^{-4}$ ), smaller than what is measured in the material identification, can also be part of the explanation.

\section{Conclusion}

The vibration behavior of entangled cross-linked fibers used as sandwich core material was studied.

First, experimental shear tests showed that for this material the shear modulus decreases with the amplitude at low shear strains.

Lagrange's equations were used to derive an analytical model of a sandwich beam in which the core presents amplitude-dependent shear properties. The expression of strain energy of the core was replaced by a more general expression of the virtual work due to strain, allowing any material behavior to be taken into account.

A sandwich beam with entangled cross-linked fibers as core material was tested. The first mode was observed for increasing load amplitude. The resulting Frequency Response Function showed a softening nonlinear behavior.

This behavior could be reproduced with the proposed model. A linearly decreasing shear modulus and linearly increasing loss factor were used to obtain the Frequency Response Function, which is consistent with the shear test results.

The effect of different parameters of the material (density, type of fiber) need to be investigated to gain a better understanding of the phenomena leading to the observed softening behavior. The sensitivity to sample size will also have to be quantified to be included in the property models. From a more general point of view, the model introduced will allow the use of more complex models to describe the vibration behavior of the materials used in sandwich beams. These models include a more precise description of the shape of stress-strain loops when they are not elliptic.

\section{References}

[1] Kerwin, E. M. J., 1959. "Damping of flexural waves by a constrained viscoelastic layer". The Journal of the Acoustical Society of America, 31, pp. 952-962.

[2] DiTaranto, R. A., 1965. "Theory of vibratory bending for elastic and viscoelastic layered finite-length beams". Journal of Applied Mechanics, 87, pp. 881-886.

[3] Mead, D. J., and Markus, S., 1969. "The forced vibration of a three-layer, damped sandwich beam with arbitrary boundary conditions". Journal of Sound and Vibration, 10, pp. 163-175. 
[4] Rao, D. K., 1978. "Frequency and loss factors of sandwich beams under various boundary conditions". Journal of Mechanical Engineering Science, 20, pp. 271-282.

[5] Fotsing, E., Sola, M., Ross, A., and Ruiz, E., 2012. "Lightweight damping of composite sandwich beams: Experimental analysis". Journal of Composite Materials.

[6] Jung, W.-Y., and Aref, A. J., 2003. "A combined honeycomb and solid viscoelastic material for structural damping applications". Mechanics of Materials, 35, pp. 831-844.

[7] Mezeix, L., Bouvet, C., Huez, J., and Poquillon, D., 2009. "Mechanical behavior of entangled fibers and entangled cross-linked fibers during compression.". Journal of Materials Science, 44(14), pp. 3652-3661.

[8] Mezeix, L., 2010. "Développement de matériaux d'âme pour structures sandwich à base de fibres enchevêtrées". $\mathrm{PhD}$ thesis, Ecole doctorale Aéronautique et Astronautique (AA).

[9] Shahdin, A., Mezeix, L., Bouvet, C., Morlier, J., and Gourinat, Y., 2009. "Fabrication and mechanical testing of glass fiber entangled sandwich beams: A comparison with honeycomb and foam sandwich beams". Composite Structures, 90, pp. 404-412.

[10] Poquillon, D., Viguier, B., and Andrieu, E., 2005. "Experimental data about mechanical behaviour during compression tests for various matted fibres". Journal of Materials Science, 40, pp. 5963-5970.

[11] Cai, C., Zheng, H., and Liu, G., 2004. "Vibration analysis of a beam with PCLD patch". Applied Acoustics, 65, pp. 1057-1076.

[12] Nashif, A. D., Jones, D. I. G., and Henderson, J. P., 1985. Vibration Damping. John Wiley \& Sons.

[13] Lakes, R. S., 2004. "Viscoelastic measurement techniques". Review of Scientific Instruments, 75, pp. 797-810.

[14] Pritz, T., 1990. "Non-linearity of frame dynamic characteristics of mineral and glass wool materials". Journal of Sound and Vibration, 136, pp. 263-274.

[15] Pritz, T., 1986. "Frequency dependance of frame dynamic characteristics of mineral and glass wool materials". Journal of Sound and Vibration, 106, pp. 161-169.

[16] Blevins, R. D., 1979. Formulas for Natural Frequency and Mode Shape. Krieger Publishing Company.

\section{Appendix A: Submatrices for the energy expressions}

$$
\begin{gathered}
\mathbf{M}_{w w}=\left(\rho_{\mathrm{b}} h_{\mathrm{b}}+\rho_{\mathrm{t}} h_{\mathrm{t}}+\rho_{\mathrm{c}} h_{\mathrm{c}}\right) B \int_{0}^{L} \mathbf{W} \mathbf{W}^{\mathrm{T}} d x \\
+\frac{\left(h_{\mathrm{t}}-h_{\mathrm{b}}\right)^{2}}{16} \rho_{\mathrm{c}} h_{\mathrm{c}} B \int_{0}^{L} \mathbf{W}_{, x} \mathbf{W}_{, x}^{\mathrm{T}} d x \\
+m_{\mathrm{s}} \mathbf{W}_{x=x_{f}} \mathbf{W}_{x=x_{f}}^{\mathrm{T}} \\
\mathbf{M}_{u_{\mathrm{b}} u_{\mathrm{b}}}=\rho_{\mathrm{b}} h_{\mathrm{b}} B \int_{0}^{L} \mathbf{U}_{\mathrm{b}} \mathbf{U}_{\mathrm{b}}^{\mathrm{T}} d x+\frac{1}{4} \rho_{\mathrm{c}} h_{\mathrm{c}} B \int_{0}^{L} \mathbf{U}_{\mathrm{b}} \mathbf{U}_{\mathrm{b}}^{\mathrm{T}} d x \\
\mathbf{M}_{u_{\mathrm{t}} u_{\mathrm{t}}}=\rho_{\mathrm{t}} h_{\mathrm{t}} B \int_{0}^{L} \mathbf{U}_{\mathrm{t}} \mathbf{U}_{\mathrm{t}}^{\mathrm{T}} d x \\
\mathbf{M}_{u_{\mathrm{b}} w}=\frac{h_{\mathrm{t}}-h_{\mathrm{b}}}{8} \rho_{\mathrm{c}} h_{\mathrm{c}} B \int_{0}^{L} \mathbf{U}_{\mathrm{b}} \mathbf{W}_{, x}^{\mathrm{T}} d x
\end{gathered}
$$




$$
\begin{gathered}
\mathbf{M}_{u_{\mathrm{t}} w}=\frac{h_{\mathrm{t}}-h_{\mathrm{b}}}{8} \rho_{\mathrm{c}} h_{\mathrm{c}} B \int_{0}^{L} \mathbf{U}_{\mathrm{t}} \mathbf{W}_{, x}^{\mathrm{T}} d x \\
\mathbf{M}_{u_{\mathrm{t}} u_{\mathrm{b}}}=\frac{1}{4} \rho_{\mathrm{c}} h_{\mathrm{c}} B \int_{0}^{L} \mathbf{U}_{\mathrm{t}} \mathbf{U}_{\mathrm{b}}^{\mathrm{T}} d x \\
\mathbf{K}_{w w}=\left(E_{\mathrm{b}} I_{\mathrm{b}}+E_{\mathrm{t}} I_{\mathrm{t}}\right) \int_{0}^{L} \mathbf{W}_{, x x} \mathbf{W}_{, x x}^{\mathrm{T}} d x+k_{\mathrm{s}} \mathbf{W}_{x=x_{f}} \mathbf{W}_{x=x_{f}}^{\mathrm{T}} \\
\mathbf{K}_{u_{\mathrm{b}} u_{\mathrm{b}}}=E_{\mathrm{b}} h_{\mathrm{b}} B \int_{0}^{L} \mathbf{U}_{b, x} \mathbf{U}_{b, x}^{\mathrm{T}} d x \\
\mathbf{K}_{u_{\mathrm{t}} u_{\mathrm{t}}}=E_{\mathrm{t}} h_{\mathrm{t}} B \int_{0}^{L} \mathbf{U}_{t, x} \mathbf{U}_{t, x}^{\mathrm{T}} d x
\end{gathered}
$$

The subscript , $x$ denotes one derivative and , $x x$ two derivatives with respect to $x$, respectively. 